Leading Article

\title{
Angina and myocardial infarction with normal coronary arteries
}

\author{
M.E. Bourke and D.L.H. Patterson
}

Cardiac Department, Whittington Hospital, Highgate Hill, London N19 5NF, UK.

\section{Introduction}

Coronary ischaemic syndromes, including chest pain suggestive of angina pectoris, and documented myocardial infarction, in the presence of angiographically normal coronary arteries, have been the subject of much attention. In contrast to atheromatous coronary artery disease the pathophysiology of such conditions is poorly understood and the mechanisms underlying myocardial ischaemia in these circumstances have been the cause of a great deal of conjecture and controversy.

\section{Myocardial infarction}

A small percentage of patients presenting with acute myocardial infarction are found to have angiographically normal epicardial coronary arteries. The prevalence is high in certain subsets of myocardial infarction patients, such as the very young, ${ }^{1}$ especially those infarcting during or following severe physical exercise, ${ }^{2}$ and in young women during pregnancy or using oral contraceptives and smoking. ${ }^{3}$ Documented myocardial infarction in the presence of normal coronary arteries has been reported in thyrotoxicosis; both true and factitious, ${ }^{4}$ temporally related to cocaine use $^{5}$ after acute exposure to carbon monoxide, ${ }^{6}$ and in patients with viral myocarditis. ${ }^{7}$ This issue of the Journal contains a case report on a multisystem vasculitis leading to myocardial infarction without evidence of coronary artery involvement. ${ }^{8}$

Amongst suggested mechanisms are defects in clotting factors and platelet activity, ${ }^{9,10}$ coronary artery spasm, ${ }^{11}$ thrombosis with rapid spontaneous lysis, ${ }^{12}$ coronary emboli ${ }^{1}$ and small vessel disease. ${ }^{13}$ In cases of chronic Chagas' heart disease, where there is parasympathetic ganglion destruction it is postulated that transitory sympathetic overdrive causes myocardial infarction. ${ }^{14}$ In these cases the coronary arteries are not only free of atheroma but indeed are larger in comparison to hypertensive and normal patients. Another cause of an extreme

Correspondence: D.L.H. Patterson, M.D., F.R.C.P.

Received: 6 September 1990 imbalance between myocardial oxygen supply and demand might be abnormal haemoglobin oxygen dissociation, as in carbon monoxide poisoning. ${ }^{6}$

Classic angina pectoris usually indicates myocardial ischaemia, secondary to obstructive coronary artery disease. It can arise when myocardial ischaemia is due not to reduction of coronary blood flow, but to other mechanisms such as increased cardiac work as in aortic stenosis; altered blood oxygen content in severe anaemia; rheological abnormalities of the blood in hypergammaglobinaemia or, as described in this issue of the Journal, due to thyrotoxicosis. ${ }^{15}$

In Europe, approximately $10 \%$ of patients referred to cardiac units for assessment of chest pain are found on diagnostic cardiac catheterization to have no angiographic evidence of significant coronary artery disease. ${ }^{16}$ It is important to appreciate that coronary angiograms only visualize coronary arteries of greater than $400 \mu \mathrm{m}$ diameter. The majority will have a non-cardiac cause for their symptoms and in many cases careful reassessment and investigation will reveal a musculoskeletal, oesophageal or psychological cause. ${ }^{17}$ It may be difficult to determine whether anxiety-related symptoms are a cause or consequence of the current chest pain. In a small minority of patients there will still be a clinical suspicion that symptoms are due to myocardial ischaemia.

\section{Coronary vasospasm}

In 1959 Prinzmetal et al. introduced the concept of epicardial artery spasm causing the specific clinical syndrome, 'variant angina', which is characterized by angina at rest with elevated ST segments on electrocardiogram (ECG) ${ }^{18}$ Now it is thought that this mechanism may play a role in other aspects of ischaemic heart disease. Spasm can be detected in angiographically normal segments, or may be superimposed on an atherosclerotic lesion 'dynamic coronary stenosis'. Unfortunately the literature has become confused because the term coronary spasm is often applied to any situation of 
increased coronary vasomotor tone. It should be confined to a situation of focal coronary constriction sufficient to cause transient coronary occlusion, which gives rise to attacks of ischaemic pain at rest.

Although various neural ${ }^{19}$ and humoral ${ }^{20,21}$ mechanisms have been proposed, the genesis of coronary spasm is as yet undefined. Maseri and his co-workers postulate a focal hyperreactivity to a variety of coronary vasoconstrictive stimuli acting on different receptors. ${ }^{22}$ It has been observed that in variant angina, coronary spasm usually occurs at the site of atheromatous plaques, ${ }^{23}$ but the fact that such plaques are common and classical variant angina rare, suggests that some additional factor other than the presence of plaque is required. In a large study of patients with chronic coronary ischaemic syndromes other than variant angina, Bertrand et al. ${ }^{24}$ showed that when stenosis existed there was no correlation between the degree of underlying stenosis and the occurrence of spasm. Bertrand could demonstrate ergonovine-induced angina in only $4 \%$ of those with chronic stable angina and $6 \%$ with old myocardial infarction. In contrast $20 \%$ of patients with recent infarction and $30 \%$ of those experiencing angina at rest developed focal coronary occlusion in response to ergonovine. It may be that in these acute situations there is a focal hyperreactivity to coronary vasoconstrictor agents.

Recent studies have shown that vasoactive compounds affect segments of the human coronary circulation differentially. ${ }^{22}$ This has implications in the pathophysiology of both focal coronary artery spasm and of 'Syndrome X', which has been suggested to be due to a more diffuse disturbance of coronary blood flow. Intracoronary injection of organic nitrates causes marked dilatation of epicardial vessels, suggesting a resting coronary motor tone. In contrast these doses have little affect on total coronary blood flow, implying little effect on intramural resistance vessels. Adenosine displays the opposite pattern, with a marked distal dilating effect but relatively minor actions on the epicardial vessels.

Studies of the effects of intracoronary infusion of various endogenous vasoactive substances, including calcitonin gene related peptide ${ }^{25}$ neuropeptide $Y{ }^{26}$ acetylcholine ${ }^{27}$ and substance $P,{ }^{28}$ point to a heterogeneity of responses within the human coronary circulation, and may allow some pharmacological intervention of coronary blood flow regulation. It is difficult to validate their relevance to the pathophysiology of conditions under consideration here.

The clinical course of patients with documented coronary artery spasm is variable. Some have a chronic course characterized by recurrent angina at rest, others develop spontaneous remission of symptoms. Many patients have a poor response to long acting nitrate therapy, and a good response to calcium antagonists. The incidence of sudden death in patients with documented coronary artery spasm is unknown. Since episodes of complete heart block and ventricular tachycardia are known to occur during coronary artery spasm, it may be that some patients have died as a result of these rhythm disturbances. Overall the prognosis of a patient with severe coronary atherosclerosis and coronary artery spasm depends upon the degree of atherosclerosis. $^{29,30}$

\section{Syndrome X}

The term Syndrome $\mathbf{X}$ was first used by $\mathrm{Kemp}^{31}$ in 1973 in an editorial discussion of a paper by Arbogast and Bourassa ${ }^{32}$ who performed pacing studies in patients with chest pain and normal coronary arteries and showed ST depression during atrial pacing. No mention was made of exerciseinduced ST segment changes in these patients. The authors designated their patient cohort as group X. The phrase was later popularized by Opherk et al..$^{33}$ The term has been widely used, but with a lack of agreed hard diagnostic criteria. This has resulted in a confusing picture as each study has used it own terms of reference, making comparisons very difficult.

Less than $1 \%$ of those who present to a cardiac unit with possible angina, and in whom a noncardiac cause has been excluded, will fulfil the diagnostic criteria of a tight, albeit empirical, definition of Syndrome $X:^{34}$ (a) typical angina on effort; (b) positive exercise test; (c) no demonstrable coronary stenosis; (d) no other obvious cause, such as hypertension, valve disease or overt cardiomyopathy. Even applying these strict criteria will not give an infallible diagnosis, since, for instance exercise testing has its limitations.

An alternative approach to the diagnosis is to use a conceptual definition, i.e. inducible ischaemia in the absence of large coronary artery stenosis or haemodynamic overload. This approach introduces practical problems because of the difficulty in demonstrating ischaemia unequivocally.

Several studies have used the demonstration of stimulation of anaerobic metabolism as evidence of ischaemia. This is a specific but not sensitive marker. In studies of patients with postulated Syndrome X, 12-100\% demonstrated myocardial lactate production in response to pacing or dipyridamole infusion. ${ }^{34,35}$

A fall in coronary sinus oxygen saturation in response to increased workload, as induced by pacing, provides an alternative indirect mark of ischaemia and indicates a level of energy consumption beyond the limits of autoregulation of flow. In normal subjects, coronary sinus oxygen saturation 
falls transiently as pacing rate is increased but returns to normal with autoregulation within 20 seconds. Crake et al. ${ }^{36}$ demonstrated that in patients with atheromatous coronary artery disease coronary sinus oxygen falls with the onset of ischaemia, as measured by pacing-induced ST segment depression, and remains low, continuing to fall with further increases in pacing rate. Ten patients with Syndrome $X$ were also studied, two of whom had responses almost identical to those of obstructive coronary artery disease. The others showed normal coronary sinus oxygen responses, raising the possibility that their pain might not reflect myocardial ischaemia.

A number of studies have attempted to evaluate left ventricular function in patients with Syndrome $X$. Arbogast and Bourassa's original paper demonstrated no change in left ventricular function during stress induced by atrial pacing in patients. ${ }^{32}$ Levy et al. ${ }^{37}$ measuring pulmonary artery diastolic pressure, demonstrated that, in contrast to the haemodynamic changes that occur during myocardial ischaemia in coronary artery disease, chest pain and ST segment changes in patients with Syndrome $X$ are not associated with impaired left ventricular function. In contrast, other studies ${ }^{38}$ show regional wall motion abnormalities, shortening of diastolic time and raised left ventricular end-diastolic pressures during stress by pacing and exercise testing.

There have been several studies demonstrating an impaired rise in coronary blood flow in response to atrial pacing in patients with Syndrome X. In Cannon's studies ${ }^{38}$ patients developing their typical chest pain during atrial pacing demonstrated significantly lower great cardiac vein flow and higher calculated coronary resistance compared with those without pacing-induced chest pain. The chest pain group also demonstrated significantly higher lactate production and abnormalities of left ventricular systolic and diastolic function suggestive of myocardial ischaemia. These and other similar studies are consistent with a hypothesis that some patients with chest pain and angiographically normal epicardial arteries have dynamic abnormalities of the coronary microcirculation. This abnormal vasodilator capacity or vasoconstriction may result in angina pectoris and myocardial ischaemia. This has been termed microvascular angina.

If Syndrome $X$ is due to small vessel disease, a pathogenesis is as yet unknown. No evidence of a structural abnormality of small arteries has ever been found, except in a small group of atypical patients who may have early cardiomyopathy. ${ }^{39}$ Conversely there is evidence that microvascular angina may give rise to cardiomyopathy. ${ }^{40}$

Studies of forearm vasodilator reserve in normotensive patients with microvascular angina,${ }^{41}$ which measured hyperaemic response to forearm ischa- emia, demonstrated significantly reduced hyperaemia and higher vascular resistance in patients with microvascular angina than in the control group. It is postulated that patients with Syndrome $\mathrm{X}$ appear to have an impairment of vasodilator reserve that affects not only the coronary circulation but also their peripheral arterial bed.

Several studies have reported an excellent prognosis for patients with angiographically normal coronary arteries, regardless of the aetiology of the chest pain. The largest study of 1,977 consecutive patients with either normal coronary arteries or 'insignificant coronary artery disease' showed a $98 \% 10$ year survival with a $2 \%$ risk of myocardial infarction at 10 years for patients with normal coronary arteries. Patients in both groups continued to have symptoms that resulted in frequent hospitalizations, medication use, and employment disability. Almost $50 \%$, in any given year of follow-up, could not perform activities of high metabolic requirement. Although these patients are at low risk of death many remain functionally impaired for years. ${ }^{42}$

In Kemp's study the 6 year survival was no different from that of an asymptomatic age and sex matched cohort. ${ }^{17}$ However, in most long term series the clinical characteristics of the patients have not been adequately described. Groups with oesophageal, musculoskeletal and psychosomatic causes have not been excluded. Opherk et al..$^{43}$ characterized a group of 40 Syndrome $X$ patients with abnormal flow responses to dipyridamole; at a mean follow-up of 4 years, all patients were alive, and symptom status was unchanged in most. No patient had a spontaneous improvement or remission of symptoms. It must be noted that 15 of these 40 patients had conduction abnormalities on their ECG during exercise, of these 5 developed persistent bundle branch block, and 6 progressed towards a dilated cardiomyopathy. It is possible that the abnormality responsible for limited coronary flow reserve in this subgroup is different from that with normal ECGs. Many patients with conduction abnormalities on ECG, at rest or during exercise, do not enjoy the same benign prognosis with respect to ventricular function.

Epidemiological studies of patients with chest pain and normal coronary arteries are reassuring with regard to mortality, but do indicate considerable long term morbidity and medical/social and economic consequences. An alternative aetiology should be sought for the chest pain by history, examination and appropriate investigation. With firm diagnosis of a non-cardiac cause these patients can be effectively managed.

Treatment of the small group of patients remaining with a diagnosis of microvascular angina is difficult. Glyceryl trinitrate is helpful for short term relief of pain in most cases. Many, but not all, 
respond to calcium channel blockers. Beta-blockers can sometimes be of benefit, possibly by reducing myocardial oxygen demand. ${ }^{44}$ Intravenous aminophylline has recently been demonstrated to increase effort tolerance, improve symptoms and abolish ischaemic ST segments during exercise in patients with Syndrome $X .^{45}$ It is suggested that a transmural steal phenomenon occurs whereby there is a maldistribution of coronary flow due to an abnormally elevated resistance upstream of the small vessels. It may be that aminophylline blockade of adenosine receptors blunts excessive arteriolar dilatation thus preventing this maldistribution. It remains to be seen whether oral aminophylline preparations can confer the same benefit. Since the condition of Syndrome $X$ has been so poorly defined it is not surprising that there have been no adequate clinical trials of treatment for the condition.

\section{References}

1. Rosenblatt, A. \& Selzer, A. The nature and clinical features of myocardial infarction with normal coronary angiogram. Circulation 1977, 55: 578-580.

2. Delaye, J., Beaune, J. \& Delchaye, J.P. Myocardial infarction at a young age during high physical exercise. In: Roskamm, H. (ed.) Myocardial Infarction at a Young Age. Springer, Berlin, Heidelberg, New York, 1981, pp. 115-221.

3. Schapiro, $S$. Oral contraceptives and myocardial infarction (editorial). N Engl J Med 1975, 293: 195-196.

4. Bergeron, G.A., Goldsmith, R. \& Schiller, N.B. Myocardial infarction; severe reversible ischaemia and shock following excess thyroid administration in a woman with normal coronary arteries. Arch Intern Med 1988, 148: 1450-1453.

5. Kossowsky, W.A., Lyon, A.F. \& Chou, S.Y. Acute non Q wave cocaine related myocardial infarction. Chest 1989, 96: 617-621.

6. Marius-Nunez, A.L. Myocardial infarction with normal coronary arteries after acute exposure to carbon monoxide. Chest 1990, 97: 491-494.

7. Miklozek, C.L., Crumpacker, C.S., Royal, H.D., Come, P.C., Sullivan, J.L. \& Ableman, W.H. Myocarditis presenting as an acute myocardial infarction. Am Heart J 1987, 115: 786-789.

8. Rajani, R.M., Dalvi, D.V., D'Silva, S.A., Lokhandwala, Y.Y. \& Kale, P.A. Acute myocardial infarction with normal coronary arteries in a case of polyarteritis nodosa; possible role of coronary artery spasm. Postgrad Med J 1990, 67: 77-79

9. Penny, W.J., Colvin, B.T. \& Brooks, N. Myocardial infarction with normal coronary arteries and factor XI deficiency. Br Heart J 1985, 53: 230-234.

10. Rapold, H.J., Haeberli, A., Kuemmerli, H., Weiss, M., Baur, H.R. \& Straub, W.P. Fibrin formation and platelet activation in patients with myocardial infarction and normal coronary arteries. Eur Heart $J$ 1989, 10: 323-323.

11. L'Abbate, A., Biagini, A. \& Mazzeri, M.G. Major role of coronary spasm in the pathogenesis of myocardial infarction at a young age. In: Roskamm, H. (ed.) Myocardial Infarction at a Young Age. Springer, Berlin, Heidleberg, New York, 1981, pp. 129-136.

12. Engel, H.J., Engle, E. \& Lichtlen, P.R. Acute myocardial infarction in women, evidence for spontaneous lysis coronary thrombus. In: Roskamm, H. (ed.) Myocardial Infarction at a Young Age. Springer, Berlin, Heidelberg, New York, 1981, pp $129-136$

13. Mason, J.W. \& Strefling, A. Small vessel disease of the heart resulting in myocardial necrosis and death despite angiographically normal coronary arteries. Am J Cardiol 1979, 44: $171-176$.

14. Bestetti, R.B., Finzi, L.A. \& Oliveira, J.S.M. Chronic Chagas' heart disease presenting as an impending acute myocardial infarction; a case of favouring the neurogenic pathogenesis complex. Clin Cardiol 1987, 10: 368-370.

15. Glickson, M., Freimark, D., Leor, R., Shechter, M., Kaplinsky, E. \& Rabinowitz, B. Unstable anginal syndrome and pulmonary oedema due to thyrotoxicosis. Postgrad Med J 1990, 67: 77-79.
16. Dart, A.M., Alban-Davies, H., Dalal, J., Ruttley, M. \& Henderson, A.H. Angina and normal coronary arteriograms: a follow up study. Eur Heart J 1980, 1: 97-100.

17. Kemp, H.D., Vokonas, P.S., Cohn, P.F. \& Gorlin, R. The anginal syndrome associated with normal coronary arteriograms. Report of a 6 year experience. Am J Med 1973, 54: 735-742.

18. Prinzmetal, M., Kennamer, R., Merliss, R., Wada, T. \& Bor, N. Angina pectoris: a variant form of angina pectoris. Preliminary report. Am J Med 1959, 27: 375-388.

19. Heusch, G. \& Guth, B.D. Neurogenic regulation of coronary vasomotor tone. Eur Heart J 1989, 10 (suppl F): 6-14.

20. Matsuyama, K., Hirofumi, Y., Okumura, K. Effects of $\mathbf{H}_{1}$ receptor stimulation on coronary arterial diameter and coronary haemodynamics in humans. Circulation 1990, 81: 65-71.

21. Moncada, S. \& Vane, J.R. Arachadonic acid metabolites and the interactions between platelets and blood vessel walls. $N$ Engl J Med 1979, 300: 1142-1145

22. Maseri, A., Newman, C. \& Davies, G. Coronary vasomotor tone: a heterogenous entity. Eur Heart J 1989, 10 (suppl F): 2-5.

23. McAlpine, $R$. Relation of coronary arterial spasm to the sites of organic stenoses. Am J Cardiol 1989, 46: 143-146.

24. Bertrand, M., La Blanche, J., Tilmant, P. et al. Frequency of provoked coronary arterial spasm in 1089 consecutive patients undergoing arteriography. Circulation 1982, 65: 1299-1306.

25. McEwen, J., Larkin, S., Davies, G. et al. Calcitonin gene related peptide: a potent dilator of human epicardial coronary arteries. Circulation 1986, 74: 1243-1247.

26. Clarke, J., Davies, G., Kerwin, R. et al. Coronary artery infusion of Neuropeptide $\mathrm{Y}$ in patients with coronary artery disease. Lancet 1987, i: 1057.

27. Newman, C., Hackett, D., Fryer, M., El Tamimi, H., Davies, G. \& Maseri, A. Dual affects of acetylcholine on angiographically normal coronary arteries. Circulation 1987, 76 (suppl 4): 56.

28. Crossman, D., Larkin, S., Fuller, R. et al. Substance P dilates epicardial vessels and increases coronary blood flow in man. Circulation 1988, 78: II-412.

29. Bott-Silverman, C. \& Heupler, F.A. Jr. What is the long term prognosis of patients with coronary spasm and normal coronary arteries? Int J Cardiol 1984, 6: 112-116.

30. Nakamura, M., Takeshita, A. \& Nose, I. Clinical characteristics associated with myocardial infarction, arrhythymias and sudden death in patients with vasospastic angina. Circulation 1987, 75: $1110-1116$.

31. Kemp, H.D. Left ventricular function in patients with anginal syndrome and normal coronary arteries. Am J Cardiol 1973, 32: 375-376.

32. Arbogast, R. \& Bourassa, M.G. Myocardial function during atrial pacing in patients with angina pectoris and normal coronary arteriograms. Am J Cardiol 1973, 32: 257-263. 
33. Opherk, D., Zebe, H., Wehie, E. et al. Reduced coronary dilatory capacity and ultrastructural changes of the myocardium in patients with angina but normal coronary angiograms. Circulation 1981, 63: 817-825.

34. Hutchison, S.J., Poole-Wilson, P.A. \& Henderson, A.H. Angina with normal coronary arteries - a review. $Q J$ Med 1988, Series 72, 268: 677-688.

35. Cannon, R.O., Leon, M.B., Watson, R.M., Rosing, D.R. \& Epstein, S.E. Chest pain and normal coronary arteries - role of small coronary arteries. Am J Cardiol 1985, 55: 50B-60B.

36. Crake, T., Crea, P.A., Shapiro, L., Canepa-Anson, C. \& Poole-Wilson, P.A. Continuous recording of coronary sinus oxygen saturation during atrial pacing in patients with angina pectoris or Syndrome X. Br Heart J 1988, 59: 31-38.

37. Levy, R.D., Shapiro, L.M., Wright, C., Mockus, L. \& Fox, K.M. Syndrome $X$ : the haemodynamic significance of ST depression. Br Heart $J$ 1986, 56: 353-357.

38. Cannon, R.O., Benow, R.O., Bachararach, S.L. et al. Left ventricular dysfunction in patients with angina pectoris, normal epicardial coronary arteries, and abnormal vasodilator reserve. Circulation 1985, 71: 218-226.

39. Masseri, M., Yarom, R., Gotsman, M.S. \& Hasseyn, Y. Histologic evidence for small vessel coronary artery disease in patient with angina pectoris and patent large coronary arteries. Circulation 1986, 74: 964-972.
40. Factor, S.M. \& Sonnenblick, E.H. The pathogenesis of clinical and experimental congestive cardiomyopathies: recent concepts. Prog Cardiovasc Dis 1985, 27: 395-420.

41. Cannon, R.O., Hansen, C. \& Epstein, S.E. Impaired forearm vasodilatory reserve in patient with microvascular angina. Evidence of a generalised disorder of vascular function? $N$ Engl J Med 1987, 317: 1366-1370.

42. Papiniconaou, M.N., Caliss, R.M., Halatky, M.A. et al. Prognostic indications of angiographically normal and insignificantly narrow coronary arteries. Am J Cardiol 1986, 58: $1181-1187$.

43. Opherk, D., Schuler, G., Wetterauer, K., Mathey, J., Schwarz, F. \& Kubler, W. Follow-up study (4 years) in patient with angina pectoris and normal coronary arteriogram: (Syndrome X). Circulation 1989, 80: 1610-1616.

44. Bugiardini, R., Borghi, A., Biagetti, L. \& Puddu, P. Comparison of verapamil v. propranolol therapy in Syndrome $X$. Am J Cardiol 1989, 63: 286-290.

45. Emdin, M., Picano, E., Lattanzani, F. \& L'Abbate, A. Improved exercise capacity with acute aminophylline administration in patients with Syndrome X. J Am Coll Cardiol 1989, 14: 1450-1453. 